Sensors 2003, 3, 451-457

sensors

ISSN 1424-8220

(C) 2003 by MDPI

http://www.mdpi.net/sensors

\title{
Semiconductor Metal Oxide Sensors in Water and Water Based Biological Systems
}

\author{
Fatima Kh. Chibirova*, Alexander K. Avetisov, Serge A. Kazakov, Ludmila A. Obvintseva and \\ Marina V. Strobkova
}

Karpov Research Institute for Physical Chemistry, Russia 1050646 Moscow, ul. Vorontsovo Pole, 10, Tel. (095) 735-65-57, Fax: (095) 437-28-10

Author to whom correspondence should be addressed: chibir@cc.nifhi.ac.ru

Received: 1 May 2003 / Accepted: 15 September 2003 / Published:31 October 2003

\begin{abstract}
The results of implementation of $\mathrm{In}_{2} \mathrm{O}_{3}$-based semiconductor sensors for oxygen concentration evaluation in water and the LB-nutrient media (15.5 g/l Luria Broth Base, Miller (Sigma, Lot-1900) and $\mathrm{NaCl}$ ) without bacteria and with E.coli bacteria before and after UV-irradiation are presented.
\end{abstract}

Keywords: semiconductor sensors, water, liquid phase biological systems

\section{Introduction}

Effective implementation of semiconductor metal oxide sensors (SMOS) for air and gas media analysis may find logical extension. There are good options to use these sensors for analysis of various gas components dissolved in polar liquids (for instance, in water or liquid phase biological systems). Such possibility results from an increase of electron transfer rate between adsorbed gas molecules and semiconductor surface, caused by polar media. That is why theoretic options exist for development of a method for gas analysis at a temperature of a liquid system. Such method could not only simplify a measurement process, but also improve sensor sensitivity as a result of increase of gas molecules adsorption rate under the conditions of temperature decrease.

Though advantages of semiconductor metal oxide sensors are explicit (i.e. high sensitivity, operating speed, small sizes, options for real time measurements, comparatively low cost), only few papers were thitherto published on the implementation of this method for gas analysis in liquid media. 
Experimental approach was presented in the series of papers of Miasnikov and co-authors [1-4], in which the semiconductor sensors technique for gas analysis in polar liquids was developed. According to these papers, the trend has been toward intensification of the processes of adsorption and desorption of gases and radical particles on the semiconductor surfaces $\left(\mathrm{ZnO}, \mathrm{TiO}_{2}\right)$ in polar liquids without additional heating, which is necessary in the case of gas media. Equations are proposed [3], which interlink sensor electrophysical parameters with oxygen concentration in a liquid.

It should be emphasised that the results in [1-4] were obtained in the sells, separated from environment, and in the low concentration range of dissolved oxygen $\left(2,24 \mathrm{E}^{-03}-4,80 \mathrm{E}^{-7} \mu \mathrm{g} / \mathrm{l}\right)$. But the most important, as a practical matter, liquid phase systems, i. e biochemical liquids, such as blood, cytoplasm and others, constitute open systems with wide range of oxygen concentrations, as compared to concentrations investigated in the above papers. That is why our understanding is that SMOS-based biosensors have good prospects of implementation for open systems at high concentrations of dissolved oxygen. We should also like to emphasise that direct extrapolation would be unfounded of the results, obtained in [1-4], to the open liquid phase systems. Special investigations should be carried out for reconciliation of the conclusions, made in [1-4], with the results, which would be obtained for the open systems at high concentration of dissolved oxygen. Bio-sensors seem to be the efficient in this direction, and special research should be carried out with the use of bio-system models for biosensors development on the basis of SMOS.

When choosing test model for our investigations we were guided by one of the most important problem of medical diagnostics of virus infections, which is connected to early virus recognition in human organism. This problem could be solved by means of assessment of the quantity of gas phase vital function products of the microorganisms (including oxygen) in a nutrient medium at a stage, that forerun active growth of the above microorganisms. With this purpose we have developed sensitive semiconductor metal oxide sensors for evaluation of micro alterations of oxygen concentrations in a nutrient medium, when it contains pathogen bacteria.

In our paper the results are presented of implementation of $\operatorname{In}_{2} \mathrm{O}_{3}$-based semiconductor sensors for oxygen concentration evaluation in the LB-nutrient media (15.5 g/l Luria Broth Base, Miller (Sigma, Lot-1900) and $\mathrm{NaCl}$ ) without bacteria and with E.coli bacteria before and after UV-irradiation.

\section{Experimental procedure}

Semiconductor sensors for measurement of dissolved oxygen concentration in the water represent insulating substrate with measurement Pt-electrodes, applied by cathodic sputtering method, and $\operatorname{In}_{2} \mathrm{O}_{3}$ sensitive semiconductor layer (width $1 \mu \mathrm{m}$ ) applied according to special technology, providing good adhesion and water resistance.

Test objects: 1) pure water (twice-deionized, twice-distilled water, transmitted through ionit and nuclear membrane with pore size of $0,5 \mu \mathrm{m}) ; 2)$ water solutions $\mathrm{NaCl}(0,1-0,8) \mathrm{g} / \mathrm{l} ; 3)$ LB-nutrient media without bacteria; 4) LB- nutrient media with bacteria E.coli.

E.coli C600-lux culture was used, which contains multy-copy plasmid with luciferase gene (luminosity range from 400 to $480 \mathrm{~nm}$ ). The cells were cultivated at $33^{\circ} \mathrm{C}$ in LB-nutrient media, produced with the following contents: $15.5 \mathrm{~g} / \mathrm{l}$ Luria Broth Base, Miller (Sigma, Lot-1900) with 
addition of $\mathrm{NaCl}$. E.coli C600-lux culture, containing multy-copy plasmid with luciferase gene with LEX-A promotor, was chosen in such a way that produced system provide oxygen self-sufficiency.

Sensor calibration was carried out in the isolated calibration cell for all water systems. Nitrogen, helium or argon was used as carrier gas for extrinsic oxygen. Gas mixtures with predetermined concentrations by means of dynamic generator of standard mixtures (ГГС-3 brand). Gas mixture was delivered to calibration cell by bubbling through glass tube with Shott filter. Output sensor response was transferred from the secondary actuator to PC through L-card interface.

\section{Results and Discussion}

Results of sensor calibration in pure water, in $\mathrm{NaCl}(0,5 \mathrm{~g} / \mathrm{l})$ water solution and in LB-nutrient media are presented in Fig 1. As it was revealed in [3], under stationary operation condition of a sensor in separated sell at low oxygen concentration, oxygen concentration dependence of electrocunductivity is as follows:

$$
\left(\sigma_{0} / \sigma_{\mathrm{m}}\right)^{2}-1=\mathrm{K} \cdot C, \quad C=\alpha \cdot p,
$$

where $\sigma_{0}$ and $\sigma_{\mathrm{m}}$ - initial (before oxygen adsorption) and stationary (after achievement of oxygen adsorption-desorption process) sensor electroconductivity, $\mathrm{K}$ - constant, which is proportional to the ratio of constants of oxygen adsorption-desorption $\left(\kappa_{1}\right.$ and $\left.\kappa_{2}\right) ; C$ - concentration of dissolved oxygen; $p$ - partial oxygen pressure over liquid surface; $\alpha$ - Bunzen factor.

Calibration curves for all three systems (Fig. 1) are described by formula1 (1) within the range of experimental error.

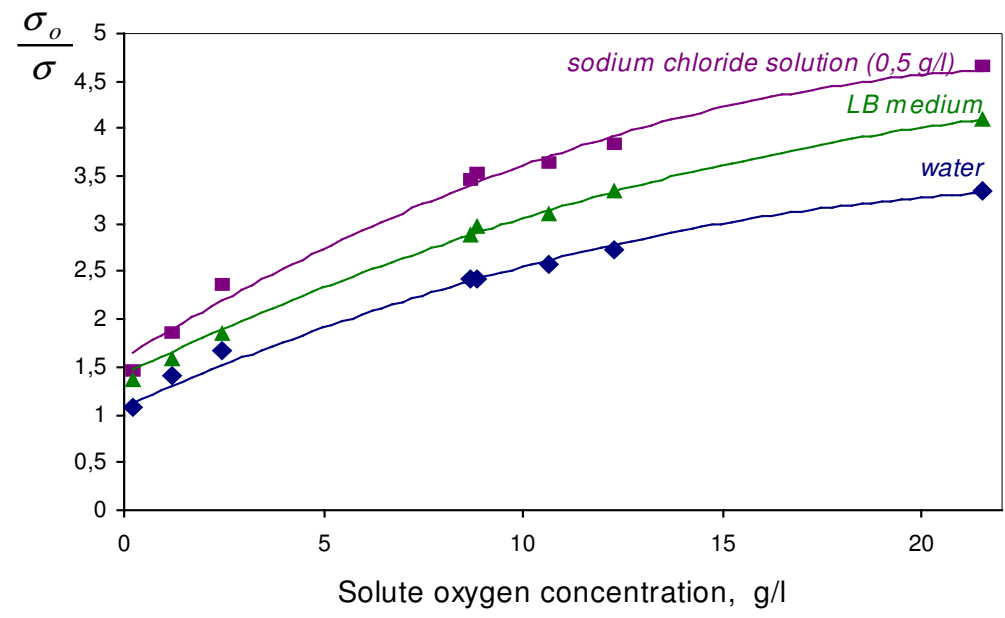

Figure 1. Calibration curves for semiconductor sensor a: in water, in $\mathrm{NaCl}$ water solution, in $\mathrm{LB}$ nutrient media.

Observed sensor sensitivity in $\mathrm{NaCl}$ solution is higher as compared to pure water (Fig. 1). The same effect, though at less extent (Fig. 1), is observed in LB-nutrient media containing $0,5 \mathrm{~g} / \mathrm{l} \mathrm{NaCl}$ and organic admixtures. We have carried out a series of experiments on low $\mathrm{NaCl}$ concentration influence on oxygen sensitivity of the sensor in an open water system. Obtained results are presented 
in Fig. 2. As it is shown in Fig. 2, the sensitivity of the sensor increases with $\mathrm{NaCl}$ concentration increase, which is in good agreement with the data of [4], which was obtained for closed cell.

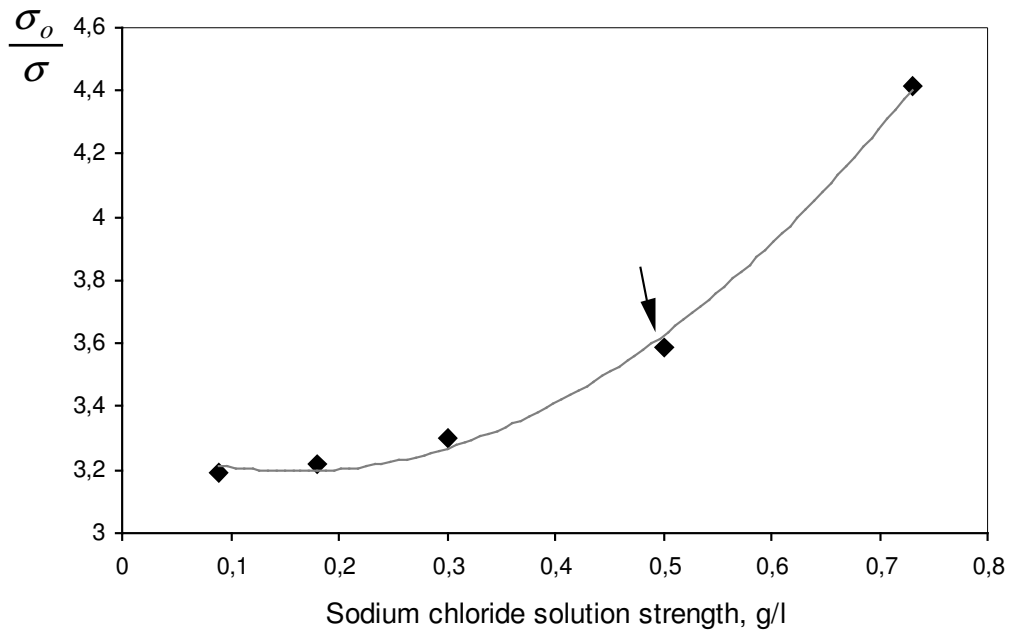

Figure 2. $\mathrm{NaCl}$ concentration dependence of semiconductor sensor. The arrow mark $\mathrm{NaCl}$ concentration in LB-nutrient media.

As it was already mentioned, the major objective of our investigation is to show that semiconductor metal oxide sensors can be successfully implemented in biological liquids for detection of micro changes of dissolved oxygen concentration, resulted from bacteria vital function at various stages of its growth. With the help of sensor we intended to reveal the dependence between dissolved oxygen concentration changes and state of bacteria, i.e. their viability level. With the purpose of changes in bacteria state they were subjected to UV-irradiation, which influenced upon their viability. Beforehand the influence of UV-irradiation upon LB-nutrient media was investigated (Fig. 3).

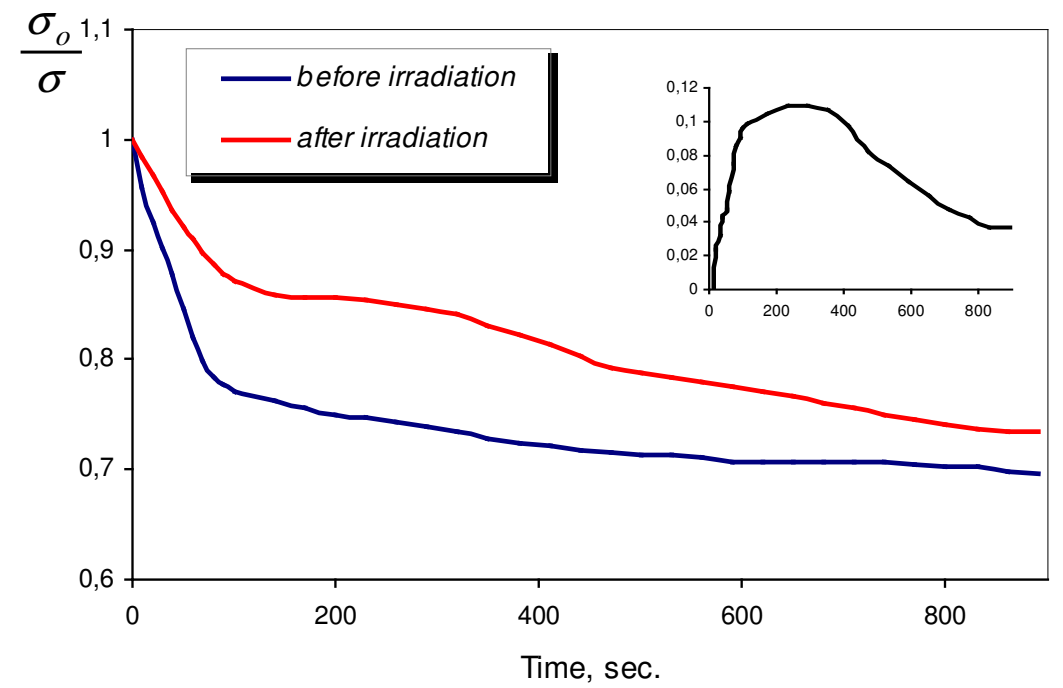

Figure 3. Kinetics of sensor response in LB-nutrient media before and after UV-irradiation. Inset shows the result of subtraction of these curves. 
It is shown that during first minutes of UV-irradiation, the kinetic of sensor response changes strongly. In the inset in Fig. 3 the result of subtraction of sensor responses before and after UVirradiation is presented. The result of subtraction may be explained as the rise of new donor signal (after UV-irradiation of LB-media) on the background of unchanged oxygen sensor response. The above donor signal completely disappears 10-15 minutes after UV-irradiation. The only possible explanation of this fact is the appearance of fragments of organic molecules with short life period in LB-media, as a result of UV-irradiation.

It is well known that E.coli cells are characterised by complicated mode of damage repair, which was elaborated during evolution process. In the case of damage in DNA molecules, resulted from various chemical or physical factors, including UV-irradiation, the SOS-lux system is being launched. This system is based upon luminescent bacteria ability to radiate visible light as a result of luciferase catalysis. This process is accompanied by oxygen absoption and is described by the below scheme:

$$
\begin{aligned}
& \text { luciferase } \\
& \mathrm{FNMH}_{2}+\mathrm{RCHO}+\mathrm{O}_{2} \rightarrow \mathrm{FNMH}+\mathrm{RCOOH}+\mathrm{H}_{2} \mathrm{O}+\mathrm{hv},
\end{aligned}
$$

where $\mathrm{FNMH}_{2}$ - unsaturated flavionic mononucleotid; RCHO - long oil polymer aldehyde; $\mathrm{RCOOH}$ long oil polymer acid.

So, after irradiation in the culture methabolic processes are launched, which are accompanied by decrease of oxygen absorption. But simultaneously the system is launched of DNA repairing in E.coli bacteria cells, which increases oxygen absorption.

With the purpose of investigation UV-irradiation influence upon change of dissolved oxygen concentration in LB-media, containing E.coli bacteria, the test specimens of bacteria culture were prepared and stored at room temperature $\left(20^{\circ} \mathrm{C}\right)$. Metabolism rate substantially depends upon temperature. Optimal temperature value is higher than $30^{\circ} \mathrm{C}$. That's why at $20^{\circ} \mathrm{C}$ the rate of cells division process is low or equals to zero. If the division process takes place, it should influence upon dissolved oxygen concentration. Slow oxygen concentration decrease was indicated by means of sensor in the test specimens of the bacteria culture (Fig. 4).

Then test specimen was placed into UV-irradiation chamber (wave length 10-400 $\mathrm{nm}$, irradiation time 3 minutes, integral flow density 250 watt-second $/ \mathrm{m}^{2}$ ). During the period of specimen irradiation, the sensor was placed into pure nutrient medium. 5 seconds after the completion of test specimen irradiation, the measurements of concentration of dissolved oxygen were started.

Fig. 4 presents kinetic curves concentration changes of dissolved oxygen in the test specimen after irradiation, which was obtained by means of semiconductor sensor. The comparison of the curves reveals that sensor response in the specimen after UV-irradiation decreases 3 times quicker than sensor response in the test specimen of the same culture before irradiation. Just this result was should be realised in case of validity of scheme 2 .

The curve of the irradiated specimen (bottom curve, Fig. 4) also presents a some peculiarity, which reveals at the same time interval as additional donor signal in the LB-pure nutrient medium (Fig. 3). So, we can assume that to parallel processes take place, and their influence upon the sensor response 


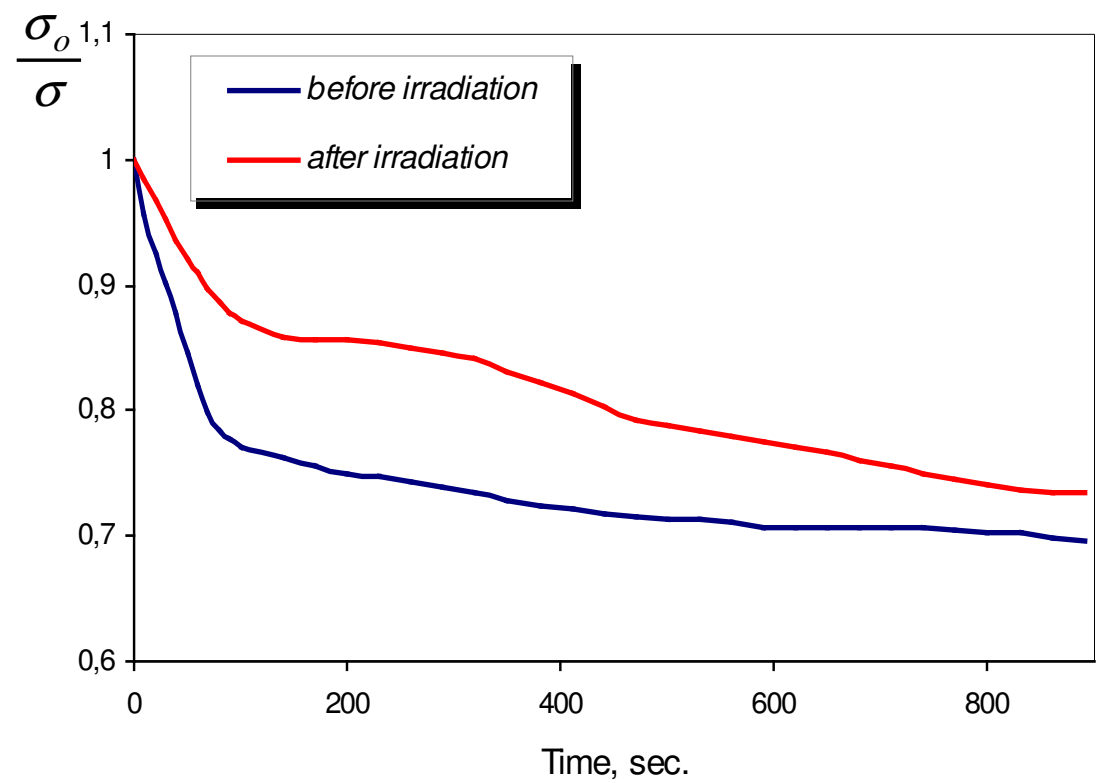

Figure 4. Kinetics of sensor response in LB-nutrient media with E.coli bacteria before and after UV-irradiation.

kinetics differs one from another. First of all, it is quick decrease of dissolved oxygen concentration in the specimen after irradiation, which results from increase of oxygen absorption by E.coli bacteria through the launch of SOS-reparation mechanism. Secondly, it is creation of high molecular radicals LB-media and their further recombination and disappearance.

\section{Conclusion}

In our research we demonstrated and proved availability of options for implementation of semiconductor metal oxide sensors (SMOS) in various liquid phase systems for evaluation of dissolved oxygen concentration changes under real time conditions. The proposed techniques present high sensitivity, which cannot be achieved by other techniques. This allows investigating kinetics of the complicated biological processes, such as bacteria sells division (transfer to the logarithmic growth phase, and cells reparation processes, which result from UV irradiation.

We can state with assurance that semiconductor sensor techniques has good prospects in the area of biological and biochemical systems, and its further development will become substantial contribution to bio-sensor science.

Authors express their gratitude to Dr. Alipov E.D. (MIFI) for his kind co-operation during arrangement of the investigations and for supply of LB-nutrient media and bacteria culture.

\section{References and Notes}

1. Myasnikov, I. A; Sisoeva, A.P. Journal Physical Chemistry, 1981, 55, 1278- 1282.

2. Myasnikov, I. A; Sisoeva, A.P. Journal Physical Chemistry, 1981, 55, 1283-1287. 
3. Myasnikov, I. A; Sisoeva, A.P Journal Physical Chemistry, 1993, 67,1517-1522.

4. Myasnikov, I. A; Sisoeva, A.P Journal Physical Chemistry, 1993, 67, 1523-1527.

5. Zavialov, S.A.; Zavialova, L.M.; Trahtenberg, L. I. and others. Evaluation of antioxidating potential of biological liquids by means of semiconductor chemical sensors. Vestnik Rossiiskoi akademii nauk, 1998, p. 55.

Sample Availability: Available from the authors.

(C) 2003 by MDPI (http://www.mdpi.org). Reproduction is permitted for noncommercial purposes. 